\title{
Probing the Adhesion Forces of Staphylococcus aureus to Central Venous Catheters by Single-Cell Force Spectroscopy
}

\author{
Gubesh Gunaratnam $^{1}$, Philipp Jung ${ }^{1}$, Jacqueline Willeit ${ }^{1}$, Christian Spengler ${ }^{2}$, Karin Jacobs ${ }^{2}$ and \\ Markus Bischoff ${ }^{1}$ \\ ${ }^{1}$ Institute for Medical Microbiology and Hygiene, Saarland University, D-66421 Homburg/Saar, \\ Germany \\ ${ }^{2}$ Experimental Physics, Saarland University, D-66123 Saarbrücken, Germany
}

Bacterial biofilms formed on implanted medical devices pose a serious risk to the patient: Especially when formed on medical devices inserted into the blood system, such as central venous catheters (CVCs), this may yield in life-threatening infections such as endocarditis or sepsis [1]. Along with exposure time within the patient, CVCs are increasingly colonized by staphylococcal species such as $S$. aureus or S. epidermidis [2]. In the past, many studies have investigated bacterial adhesion on CVCs by determining the number of adherent cells per catheter segment, and analyzed the adhesion to host plasma protein-coated surfaces under flow conditions $[3,4]$. The exact forces with which $S$. aureus adheres to the implanted material remain unknown yet.

In order to address this question, single-cell force spectroscopy (SCFS) was used here to determine the adhesion forces of $S$. aureus to CVCs in absence and presence of host factors (cf. Figure 1). Cantilevers were functionalized with single, viable $S$. aureus cells and probed with CVC fragments (tip and external tubing sites) that were either left untreated or were pre-incubated with human plasma to mimic the situation seen in vivo, in which CVC surfaces are decorated with plasma proteins within seconds after the insertion of the device into the bloodstream. These studies demonstrated that $S$. aureus adheres to naive CVC fragments with forces that are in the lower $\mathrm{nN}$ range (4$6 \mathrm{nN}$ for tips, and 2-7 $\mathrm{nN}$ for tubings). Retraction curves indicated that adhesion of S. aureus to this kind of surface was primarily driven by hydrophobic interactions between macromolecules on the bacterial cell surface and the hydrophobic surface of this implant material. However, this dramatically changed when CVC fragments were pre-coated with blood plasma factors. Under these conditions, adhesion forces dropped down to $0.5 \mathrm{nN}$ or lower for both, tips and tubes, probably due to the decoration of the implant material with human serum albumin, the major proteinaceous factor in plasma, for which $S$. aureus possesses a low binding capacity. Marked alterations were also observed for the retraction curves, which no longer displayed the characteristic sawtooth-like profile indicative of parallel and simultaneous stretching of hydrophobic cell-wall proteins on the implant material. Instead, retraction curves more similar to those seen with $S$. aureus on hydrophilic surfaces were observed, suggesting that adhesion of the bacterium to the pre-coated surface was mainly driven by the specific interaction of a small number of bacterial cell-wall macromolecules with selected host factors that have been deposited on the surface of the implant material.

These findings indicate that the primary adhesion capacity of $S$. aureus to implant material can be significantly reduced by modifying the surface of the implant material. Pre-coating catheters with serum albumin, a procedure already suggested to reduce the formation of thrombi on CVCs, might also serve to reduce the colonization of the implant material with this potentially life-threatening bacterium [5].

\section{References:}

[1] LA Mermel et al, Clin Infect Dis 49 (2009), p. 1.

[2] M Gominet et al, APMIS 125, p. 365.

[3] KG Kristinsson, J Med Mibrobiol 28 (1989), p. 249.

[4] B Shenkman et al, Infect Immun 69 (2001), p. 4473. 
[5] The authors acknowledge funding from the Deutsche Forschungsgemeinschaft (DFG) within the collaborative research center SFB 1027.

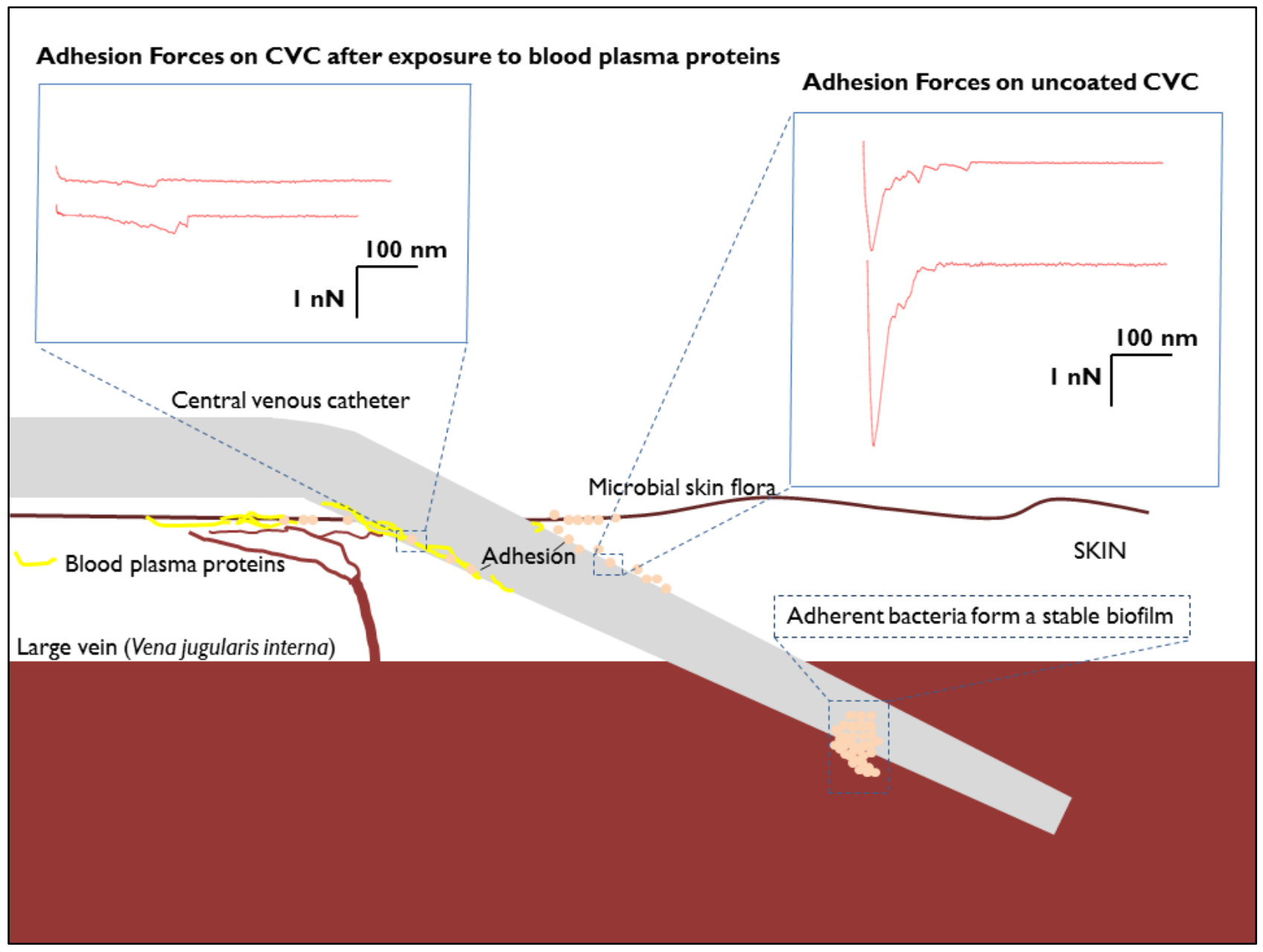

Figure 1. Illustration of catheter surface adhesion by S. aureus and force-distance curves generated with Atomic Force Microscopy. During insertion of the central venous catheter (CVC) through the skin into one of the larger veins of the upper body part (red rectangle), the surface of the CVC might be adsorbed with host proteins (yellow fibers). Some parts might also be free from protein adsorption, thus possible adhesion events and adhesion forces of skin bacteria will dependent on the occasional niches they will meet. Single Bacterial Probes and Atomic Force Microscopy revealed weak and strong adhesion forces on protein-coated and uncoated catheter surfaces, respectively (inserts). The dimension of the skin has been decreased compared to the size of the vein for illustrating reasons. 